\title{
INFLUÊNCIA DA NICOTINA DURANTE A GESTAÇÃO E LACTAÇÃO NA CICATRIZAÇÃO DA PAREDE ABDOMINAL DE RATOS LACTENTES: ESTUDO TENSIOMÉTRICO
}

\author{
Nicotine effects during pregnancy and lactation on abdominal wall healing of \\ young offspring rats: tensiometric study
}

\author{
Marcelo Ribas ALVES, Antônio Carlos Ligocki CAMPOS, Sérgio Ossamu IOSHII, \\ Hugo MORAES-JUNIOR, Danielle SAKAMOTO, Lucas Wagner GORTZ
}

ABCDDV/652

Alves MR, Campos ACL, Ioshii SO, Moraes-Jr H, Sakamoto D, Gortz LW. Influência da nicotina durante a gestação e lactação na cicatrização da parede abdominal de ratos lactentes: estudo tensiométrico. ABCD Arq Bras Cir Dig 2009;22(2):110-4

RESUMO - Racional - O tabagismo na gestação acarreta prejuízos, devidamente reconhecidos e relatados, para o crescimento intra-uterino da criança. Objetivo - Avaliar a influência da nicotina, em relação à tensiometria (tensão, força máxima e força de ruptura), na cicatrização da parede abdominal de ratos lactentes nascidos e amamentados por ratas que receberam nicotina durante a gestação e lactação. Método - Ratas Wistar prenhas foram divididas aleatoriamente em dois grupos. Um recebeu nicotina (GN) via subcutânea na dose diária de $2 \mathrm{mg} / \mathrm{kg}$ de peso durante a gestação e lactação $\mathrm{O}$ outro, controle (GC) recebeu solução fisiológica a $0,9 \%$ isovolumétrica, no mesmo período e via. Foram utilizados 60 ratos filhotes, divididos igualmente em dois grupos. Após o término do período de lactação (21 dias), todos foram submetidos à laparotomia transversa e ao fechamento da camada músculoaponeurótica. Cada grupo foi dividido em dois subgrupos, com 15 animais cada, de acordo com o intervalo de tempo utilizado para a avaliação. As aferições foram realizadas no $7^{\circ}$ (subgrupos $\mathrm{C} 7$ e N7) e no $21^{\circ}$ dia (subgrupos $\mathrm{C} 21$ e N21) do pós-operatório, quando os animais foram sacrificados. Foram retirados os retalhos da parede abdominal contendo a linha de sutura, os quais tiveram avaliação tensiométrica computadorizada. A estatística foi baseada no modelo ANOVA, ao nível de significância de 5\%. Resultados - A avaliação tensiométrica do subgrupo N7 comparada ao C7 demonstrou redução da força máxima $(0,391 \pm 0,032 \mathrm{kgf}$ vs. $0,586 \pm 0,041 \mathrm{kgf}, P=0,0060)$, da força de ruptura $(0,393 \pm 0,032 \mathrm{kgf}$ vs. $0,577 \pm 0,051 \mathrm{kgf}, P=0,0047) \mathrm{e}$ da tensão $(0,029 \pm 0,002 \mathrm{kgf} / \mathrm{mm} 2$ vs. $0,036 \pm 0,005 \mathrm{kgf} / \mathrm{mm} 2, P=0,0493)$. Entre o subgrupo N21 e C21, observou-se a diminuição da força de ruptura $(0,421 \pm 0,004 \mathrm{kgf}$ vs. $0,581 \pm 0,002 \mathrm{kgf}, P=0,0007)$ e da tensão $(0,053 \pm 0,002 \mathrm{kgf} / \mathrm{mm} 2$ vs. $0,066 \pm 0,002 \mathrm{kgf} / \mathrm{mm} 2, P=0,0056)$. Conclusão - Observouse efeito deletério da nicotina no período de gestação e lactação na cicatrização dos filhotes, de acordo com parâmetros tensiométricos.

DESCRITORES - Cicatrização. Gestação. Lactação. Laparotomia. Nicotina. Tensiometria.

\section{INTRODUÇÃO}

O Instituto Nacional do Câncer (INCA) estima que um terço dos brasileiros adultos fumem, e aproximadamente 11,2 milhões deles, são mulheres. Noventa por cento delas tornam-se dependentes em idade jovem, e a taxa de incidência de fumantes é alta nas idades entre 20 e 49 anos ${ }^{1}$.

O tabagismo na gestação acarreta prejuízos, devidamente reconhecidos e relatados, para o crescimento intrauterino da criança. Na década de 90 , do total de gestantes, $33,5 \%$ delas eram fumantes ${ }^{7}$. Assim como na gestação, as consequências do tabagismo materno sobre a lactação e a criança amamentada constituem somatório de efeitos prejudiciais ao desenvolvimento do lactente.

Dentre os vários componentes do tabaco que interferem na evolução da gravidez, destacam-se a ação da nicotina e do monóxido de carbono ${ }^{10,13,20}$. A nicotina é o principal componente vasoativo da fumaça do tabaco, inodora e

Trabalho realizado no Programa de Pós-Gradução em Clínica Cirúrgica da Universidade Federal do Paraná, Curitiba, PR, Brasil.

Endereço para correspondência: Marcelo Ribas Alves, e-mail: marcelo@criancasegura.org.br incolor, e quando inalada ou injetada pode liberar catecolaminas e resultar em vasoconstrição e diminuição da perfusão tecidual ${ }^{5}$.

Vários estudos na literatura investigaram os efeitos da nicotina sobre o processo de cicatrização. Em 1977, Mosely e Finseth foram os autores que inicialmente relataram os efeitos indesejáveis da nicotina na cicatrização tecidual, observando retardo no processo cicatricial de ferida na mão de um fumante ${ }^{15}$.

Diante dos estudos realizados para avaliar o efeito da nicotina na cicatrização, observa-se que a influência do tabagismo materno, durante a gestação e lactação sobre a cicatrização de feridas em lactentes, não foi devidamente avaliada. Portanto, são necessários estudos complementares que poderão contribuir para o conhecimento deste importante tema.

O objetivo do presente estudo é avaliar, experimentalmente, a influência da nicotina, em relação à tensiometria (tensão, força máxima e força de ruptura), no processo de cicatrização da parede abdominal de ratos lactentes nascidos e amamentados por ratas que receberam nicotina, durante a gestação e lactação. 


\section{MÉTODO}

Foram utilizados 90 ratos Wistar, adultos, com 90 dias de vida, sendo 45 machos e 45 fêmeas, formando casais aleatoriamente, acasalados na proporção de 1:1. Do total de 45 fềmeas, todas nulíparas, com peso médio de $260 \mathrm{~g}, 35$ delas engravidaram, sendo 19 ratas pertencentes ao grupo nicotina (GN) e 16 ao grupo controle (GC). Selecionaramse, de modo aleatório, quatro ratas do GN com 43 filhotes no total, e outras quatro do GC, totalizando 44 filhotes. Os animais receberam ração balanceada padrão e água ad libitum.

Nos do GN foi administrada nicotina, por via subcutânea, na dosagem de $2 \mathrm{mg}$ por $\mathrm{kg}$ de peso dia, dividida em duas doses de $1 \mathrm{mg} / \mathrm{kg}$ de peso cada (12/12 horas), diluída em $0,3 \mathrm{~mL}$ de solução fisiológica a $0,9 \%$, ajustada para $\mathrm{pH} 7,4$. Nos animais do GC foi aplicada solução fisiológica a $0,9 \%$, isovolumétrica, duas vezes ao dia (12/12 horas), por via subcutânea e sendo ajustada para pH 7,4. Administrou-se tanto a nicotina (GN) quanto a solução fisiológica (GC) a partir do $2^{\circ}$ dia de prenhez, durante todo o período gestacional e até 21 dias da amamentação dos filhotes. Ambas as doses foram ajustadas de acordo com o peso, quando necessário, até imediatamente após o parto e até o final do período de amamentação.

Os recém-nascidos foram amamentados pelas respectivas mães, desmamados no $21^{\circ}$ dia de vida e submetidos à operação imediatamente após o desmame. Foram selecionados, aleatoriamente, um total de 60 filhotes.

A técnica cirúrgica consistiu, em todos os grupos, de incisão transversa supra-umbilical envolvendo todos os planos da parede abdominal, com $30 \mathrm{~mm}$ de extensão, iniciando-se à direita e cruzando a linha média.

A diérese dos planos cuticular, aponeurótico, muscular e peritoneal iniciou-se com incisão de cinco $\mathrm{mm}$ a bisturi, realizada na borda lateral da laparotomia transversa. Procedeu-se, então, a ampliação da incisão com o uso de tesoura de íris no mesmo sentido da incisão da pele, totalizando abertura de $30 \mathrm{~mm}$ em todos os planos da parede abdominal.

Foi realizada sutura contínua não-ancorada, em bloco, interessando a aponeurose e musculatura, utilizando-se fio multifilamentar de poliglactina 4-0. Em pele, sutura subcuticular contínua não-ancorada com fio multifilamentar de poliglactina 7-0. A ferida cirúrgica foi mantida sem curativo.

Os grupos $\mathrm{C}$ e $\mathrm{N}$ foram divididos aleatoriamente em quatro subgrupos, contendo 15 animais cada, segundo a solução aplicada nas mães durante a gestação e amamentação: animais mortos no $7^{\circ}$ dia de pós-operatório, foram divididos em $\mathrm{C} 7$ e $\mathrm{N} 7$, e os no $21^{\circ}$ dia divididos em $\mathrm{C} 21$ e N21.

Removeu-se a parede abdominal em bloco e a cicatriz cirúrgica foi dividida em fragmentos contendo a cicatriz com $25 \mathrm{~mm}$ de comprimento e $30 \mathrm{~mm}$ de largura, do qual retirou-se a pele e o tecido celular subcutâneo, mantendo-se o fio de sutura da aponeurose e musculatura, encaminhadose os espécimes para estudo de resistência à tração.

De cada um desses fragmentos foi preparado um corpo de prova músculo-aponeurótico com recortes laterais na região da linha de sutura, segundo técnica descrita por $\mathrm{Nash}^{9}$ e Knolmayer et al. ${ }^{16}$. A região da linha de sutura foi medida em milímetros para o cálculo da área de secção.

Os corpos de prova foram acondicionados em geladeira térmica e encaminhados de imediato para a análise a fresco, no sentido transverso à linha de sutura, em máquina de tração computadorizada marca EMIC (Equipamentos e Sistemas de Ensaio, São José dos Pinhais, Paraná) modelo DL - $500 \mathrm{MF}$, com garras autotravantes e capacidade para $500 \mathrm{kgf}$. A célula de carga utilizada foi para $10 \mathrm{~kg}$ e a sensibilidade de 50 gramas. A velocidade da tração utilizada foi de $100.000 \mathrm{~mm} / \mathrm{min}$. A temperatura ambiente foi mantida constante em $24^{\circ} \mathrm{C}$, com umidade relativa do ar em $50 \%$.

Os parâmetros avaliados foram: força máxima, definida como a força máxima suportada pelo tecido antes do início de sua ruptura; força de ruptura, que é a força requerida para romper completamente um tecido ou parede sem considerar suas dimensões; e tensão, que é medida em termos de quantidade de força suportada por unidade de área de secção, a qual é dependente das dimensões do tecido e permite a comparação mais adequada entre corpos de prova retirados de espécimes diferentes, podendo ser mensurada em libras/ polegada ${ }^{2}$, quilogramas $/ \mathrm{m}^{2}$, quilogramas $/ \mathrm{mm}^{2}$ (Figura 1).

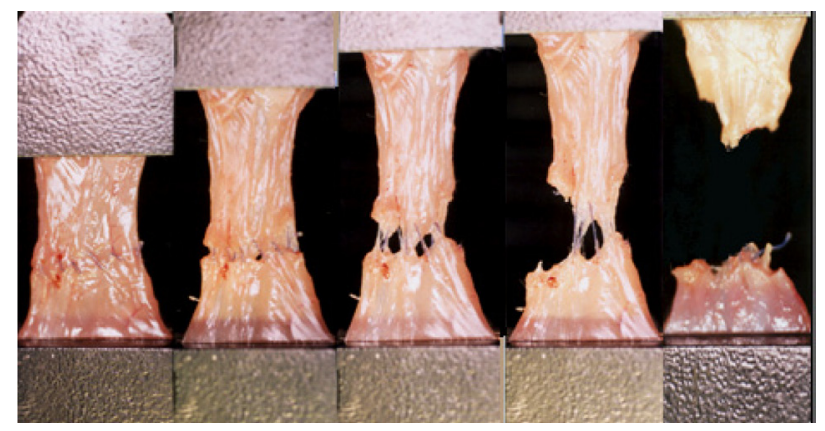

FIGURA 1 - Sequência de imagens de um corpo de prova submetido ao teste de tração

O estudo estatístico foi realizado em oito ratas, quatro do GC e outras quatro do GN, e nos 60 filhotes selecionados aleatoriamente do GC e do GN, sendo 30 pertencentes ao $\mathrm{GC}$, subdivididos em grupos $\mathrm{C} 7$ e grupo $\mathrm{C} 21$, e os outros 30 ao GN, subdivididos em grupos N7 e N21.

A análise das mães foi efetuada através do teste $t$ de Student para comparar os desempenhos médios dos dois grupos $\mathrm{C}$ e $\mathrm{N}$ com relação às características medidas nas mães nos períodos de gestação e lactação, assim como o número de filhotes nascidos em cada grupo.

Em relação às crias, a análise foi efetuada através do teste de Fisher-Snedecor. Comparou-se a variação do peso na lactação e cotejaram-se os dados da tensiometria dos corpos de prova. Considerando-se a estrutura hierárquica dos dados, seleção de mães e depois seleção de filhotes de cada mãe escolhida, com medidas ao longo do tempo (dados longitudinais) a modelagem mais apropriada foi a de Modelos Lineares Hierárquicos Condicionais (ANOVA), ao nível de significância de 5\%. 


\section{RESULTADOS}

A média do peso inicial das ratas gestantes do GN foi semelhante a do peso inicial das ratas do GC $(265,50 \pm$ $8,70 \mathrm{~g}$ vs. $259,50 \pm 21,46 \mathrm{~g}, P=0,6228)$. Os grupos completaram o período gestacional com média de peso final similar $(368,00 \pm 10,42 \mathrm{~g}$ vs. $376,50 \pm 20,87 \mathrm{~g}, P=0,4937)$.

Na lactação, as ratas do GN apresentaram média de peso final similar às do $\mathrm{GC}(310,50 \pm 18,57 \mathrm{~g}$ vs. $317,50 \pm$ $26,74 \mathrm{~g}, P=0,6822)$. Na comparação da média do ganho de peso, também houve semelhança entre os grupos $(33,50 \pm$ $17,29 \mathrm{~g}$ vs. $38,50 \pm 15,15 \mathrm{~g}, P=0,6788)$.

A comparação da média de peso, no período de lactação, dos 30 filhotes do GN e de outros 30 do GC foi: ao nascimento $P=0,4544$; aos 7 dias de vida $P=0,3447$; aos 14 dias $P=0,6056$; no desmame $P=0,4264$; no ganho de peso do período total da lactação $P=0,475$.

Para os animais sacrificados no $7^{\circ}$ dia de pós-operatório, a força máxima média foi menor para o $\mathrm{GN}$ comparado com o GC $(0,391 \pm 0,032 \mathrm{kgf}$ vs. $0,586 \pm 0,041 \mathrm{kgf}, P=0,0060)$ (Tabela 1 e Figura 2).

TABELA 1 - Médias das variáveis de força (kgf) e tensão (kgf/ $\mathrm{mm}^{2}$ ) dos subgrupos $\mathrm{C} 7, \mathrm{~N} 7, \mathrm{~N} 21$ e N21

\begin{tabular}{llcl}
\hline & & Medida \\
Variável & Grupo $(\mathbf{N})$ & Média $\pm \mathbf{E P}$ & Valor de P \\
\hline $\begin{array}{l}\text { Força Máxima } \\
(\mathrm{kgf})\end{array}$ & C7 $(15)$ & $0,568 \pm 0,011$ & 0,0060 \\
& N7 (15) & $0,391 \pm 0,011$ & \\
& C21 (15) & $1,111 \pm 0,015$ & 0,0952 \\
& N21 (15) & $1,051 \pm 0,015$ & \\
Força de Ruptura & C7 (15) & $0,577 \pm 0,009$ & 0,0047 \\
$($ kgf) & N7 (15) & $0,393 \pm 0,009$ & \\
& C21 (15) & $0,581 \pm 0,003$ & 0,0007 \\
& N21 (15) & $0,421 \pm 0,003$ & \\
Tensão & C7 (15) & $0,036 \pm 0,001$ & 0,0493 \\
$\left(\mathrm{kgf} / \mathrm{mm}^{2}\right)$ & N7 (15) & $0,029 \pm 0,001$ & \\
& C21 (15) & $0,066 \pm 0,007$ & 0,0056 \\
& N21 (15) & $0,053 \pm 0,007$ & \\
\hline
\end{tabular}

Nota: $\mathrm{EP}=$ Erro padrão

C7 e C21= Controle 7 e 21 dias, N7 e N21= Nicotina 7 e 21 dias

$\mathrm{O}$ valor $\mathrm{P}$ em negrito indica a rejeição da hipótese nula no nível de significância de $5 \%$.

Utilizou-se o teste de Fisher-Snedecor

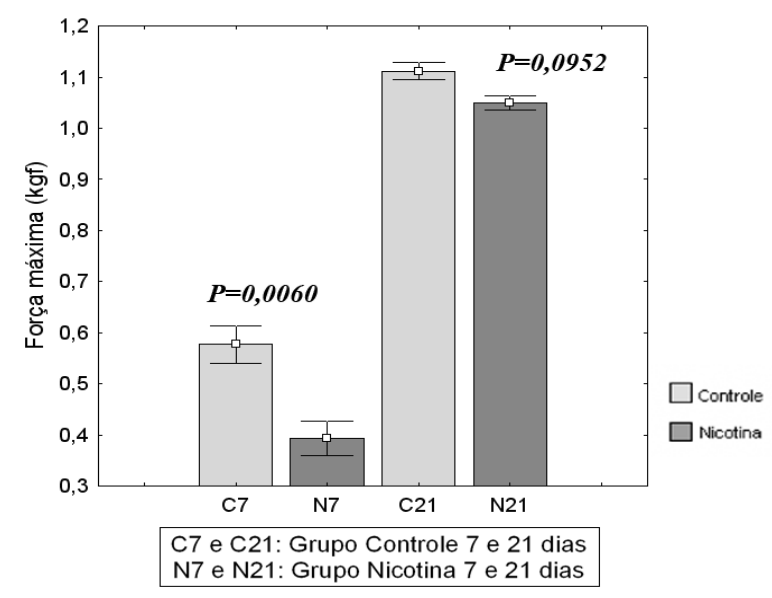

FIGURA 2 - Médias \pm DP da força máxima dos subgrupos C7, N7, C21 e N21 (kgf)
A média da força máxima dos animais sacrificados no $21^{\circ}$ dia de pós-operatório não demonstrou diferença entre o $\mathrm{GN}$ e o $\mathrm{GC}(1,051 \pm 0,013 \mathrm{kgf}$ vs. $1,111 \pm 0,022 \mathrm{kgf}$, $P=0,0952$ ) (Tabela 1).

Para o subgrupo sacrificado no $7^{\circ}$ dia, a força de ruptura média foi menor para o $\mathrm{GN}$ em relação ao $\mathrm{GC}(0,393$ $\pm 0,032 \mathrm{kgf}$ vs. $0,577 \pm 0,051 \mathrm{kgf}, P=0,0047$ ). (Tabela 1 ).

Para o subgrupo sacrificado no $21^{\circ}$ dia, a força de ruptura média foi menor para o $\mathrm{GN}$ em relação ao $\mathrm{GC}(0,421 \pm 0,004 \mathrm{kgf}$ vs. $0,581 \pm 0,002 \mathrm{kgf}, P=0,0007$ ). (Tabela 1 e Figura 3 ).



FIGURA 3 - Médias \pm DP da força de ruptura dos subgrupos C7, N7, C21 e N21 (kgf)

A tensão média, para os animais sacrificados no $7^{\circ}$ dia, foi menor para o GN comparado com o GC $(0,029$ $\pm 0,002 \mathrm{kgf} / \mathrm{mm}^{2}$ vs. $\left.0,036 \pm 0,005 \mathrm{kgf} / \mathrm{mm}^{2}, P=0,0493\right)$. (Tabela 1 e Figura 4).

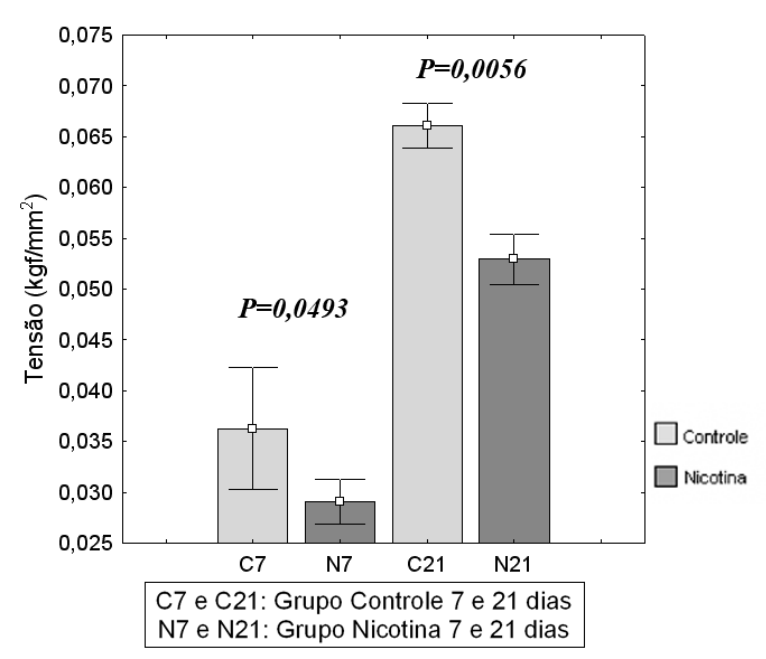

FIGURA 4 - Médias \pm DP da tensão dos subgrupos C7, N7, C21 e N21 (kgf/ $\left.\mathrm{mm}^{2}\right)$

Para os animais sacrificados no $21^{\circ}$ dia, a tensão média foi menor para o GN comparado com o GC $(0,053$ $\pm 0,002 \mathrm{kgf} / \mathrm{mm}^{2}$ vs. $\left.0,066 \pm 0,002 \mathrm{kgf} / \mathrm{mm}^{2}, P=0,0056\right)$ (Tabela 1). 
Comparando-se os resultados obtidos entre os animais do grupo controle $\mathrm{GC}$, sacrificados no $7^{\circ}$ dia (C7) e no $21^{\circ}$ dia (C21), assim como entre os do grupo nicotina (GN), sacrificados no $7 .^{\circ}$ dia (N7) e no $21 .^{\circ}$ dia (N21), observou-se aumento da média da força máxima do $\mathrm{C} 7$ para $\mathrm{C} 21(0,586$ $\pm 0,041 \mathrm{kgf}$ vs. $1,111 \pm 0,022 \mathrm{kgf}, P<0,0001)$ e do N7 para N21 $(0,391 \pm 0,032 \mathrm{kgf}$ vs. $1,051 \pm 0,013 \mathrm{kgf}, P=0,039)$.

Constatou-se também que os valores da média de força de ruptura aumentaram do C7 para C21 $(0,577 \pm 0,051 \mathrm{kgf}$ vs. $0,581 \pm 0,002 \mathrm{kgf}, P<0,0001)$ e do N7 para N21 $(0,393$ $\pm 0,032 \mathrm{kgf}$ vs. $0,421 \pm 0,004 \mathrm{kgf}, P=0,0484$ ).

Relativo à média da tensão, houve aumento do $\mathrm{C} 7$ para $\mathrm{C} 21\left(0,036 \pm 0,005 \mathrm{kgf} / \mathrm{mm}^{2}\right.$ vs. $0,066 \pm 0,002 \mathrm{kgf} / \mathrm{mm}^{2}$, $P<0,0001)$, bem como do N7 para N21 $(0,029 \pm 0,002 \mathrm{kgf} /$ $\mathrm{mm}^{2}$ vs. $\left.0,053 \pm 0,002 \mathrm{kgf} / \mathrm{mm}^{2}, P<0,0001\right)$.

\section{DISCUSSÃO}

A utilização de animais de ambos os sexos no estudo, pelo tempo máximo de 42 dias de vida, foi embasada no estudo de Hughes e Tanner, que compararam as variáveis antropométricas desde o nascimento até a vida adulta, e concluíram não haver diferenças relacionadas ao sexo antes dos 50 dias de vida para a variável peso 8 .

A utilização da parede abdominal para o estudo foi escolhida pela importância do fechamento deste segmento nas laparotomias, em especial as transversas por serem o tipo de incisão mais utilizado em recém-nascidos e crianças. É um tipo de incisão anatômica que segue as linhas de força da pele.

Com relação à técnica utilizada no fechamento, contínua ou interrompida, optou-se neste estudo pela sutura contínua $^{19}$. Outro fator de importância é o tipo de fio a ser utilizado. A aplicação de fios de sutura interfere no processo de cicatrização. O ideal é aquele que produz a menor reatividade biológica possível. Há evidências de que os fios monofilamentares inabsorvíveis provocam menor reação tecidual e, como consequência, menor interferência no processo cicatricial ${ }^{2,21}$. Optou-se pelo fio multifilamentar de poliglatina por ser o tipo de material com o qual habitualmente é feito o fechamento das laparotomias no plano músculo-aponeurótico em humanos.

A via subcutânea de injeções diárias de nicotina demonstrou ser bem tolerada pelos animais e com rápida absorção da droga, com níveis plasmáticos detectados em cinco minutos ${ }^{3}$.

A dose utilizada neste estudo ( $2 \mathrm{mg}$ por $\mathrm{kg}$ de peso em duas administrações diárias) foi baseada em amostras plasmáticas de humanos tabagistas crônicos. Segundo Richardson et al. ${ }^{18}$, é equivalente ao consumo de 60 cigarros ao dia1.

Forrest, Pang e Lindsay ${ }^{4}$ concluíram que o tabagismo influencia na cicatrização, quando o mesmo não cessa por pelo menos duas semanas antes da operação.

$\mathrm{O}$ efeito da exposição materna à nicotina, por via subcutânea durante o período gestacional e de lactação, foi avaliado quanto ao desenvolvimento pulmonar no período neonatal, e concluiu-se que nos filhotes cujas mães foram expostas à nicotina, houve diminuição do número de cap- ilares dos septos alveolares ${ }^{13}$.

Quanto ao tempo de exposição à droga, para se mensurar com total segurança, utilizou-se o tempo de gestação médio dos ratos, a partir da constatação da prenhez e durante todo o período de lactação (21 dias), de tal modo que os filhotes estiveram expostos via placentária e pela lactação com doses similares e contínuas de nicotina.

A cicatrização ideal de uma ferida está relacionada com a proliferação fibroblástica e deposição de colágeno e outros elementos do tecido conectivo. A fibroplasia e a síntese do colágeno iniciam-se nas primeiras 24 horas do ferimento, seguindo-se em 48 a 72 horas pela migração endotelial. Ao redor do $4^{\circ}$ dia há a produção de fatores de crescimento que estimulam tanto a fibroplasia como a neovascularização ${ }^{17}$. A força tênsil está relacionada com a multiplicação e maturação dos fibroblastos. Segundo Harvey $^{6}$, é necessário período de aproximadamente quatro dias para a maturação dos fibroblastos.

Os intervalos utilizados para avaliar a tensiometria foram 7 e 21 dias após o procedimento operatório. Dados da literatura relatam que a boa cicatrização de uma ferida, com desenvolvimento de força tênsil adequada, está intimamente relacionada à proliferação e deposição do colágeno e outros elementos do tecido conectivo.

Lindstedt e Sandblom ${ }^{11}$ avaliaram a tensão das cicatrizes em humanos e a compararam com a de outros animais. Concluíram que em porcos e coelhos a tensão é maior do que a dos humanos, sendo equivalente nos ratos, o que permite que estes sejam utilizados para estudos experimentais e seus resultados transferidos para o uso prático em humanos.

Para os animais mortos no $7^{\circ}$ dia de pós-operatório, os valores da força máxima $(P=0,0060)$, força de ruptura $(P=0,0047)$ e tensão $(P=0,0493)$ mostraram- se menores para os animais do GN.

Para os animais sacrificados no $21^{\circ}$ dia, os valores de força de ruptura $(P=0,0007)$ e tensão $(P=0,0056)$ foram menores para os animais do GN. A variável força máxima no $21^{\circ}$ foi semelhante entre o GC e o GN.

Esses resultados demonstram que o uso crônico da nicotina, durante a gestação e lactação em ratos, associa-se à redução da força tênsil de laparotomias transversas nos filhotes.

Quando foram comparados os momentos 7 e 21 dias, observou-se aumento de todas as variáveis tensiométricas (força máxima, força de ruptura e tensão) nos grupos controle e nicotina, porém as variáveis do grupo nicotina mantiveram-se com valores inferiores ao controle. Outros autores observaram resultados similares. Em estudo sobre o efeito da nicotina na anastomose intestinal de ratos adultos, observou que no $28^{\circ}$ dia de pós-operatório houve diminuição da força de ruptura ${ }^{18}$. Avaliou-se a tensiometria em estudo sobre o efeito da nicotina na cicatrização da parede abdominal em ratos adultos, concluiu que no $14^{\circ}$ dia há diminuição da força máxima. Medeiros et al. ${ }^{14}$ também concluíram em sua pesquisa que a nicotina exerce efeito deletério sobre a parede abdominal. Há diminuição da resistência à tensão no $10^{\circ}$ dia, assim como decréscimo da pO2 do líquido tecidual próximo à área da ferida.

Portanto, a nicotina utilizada em ratas na dose de $2 \mathrm{mg} /$ 
kg de peso ao dia, durante a gestação e lactação, influencia na cicatrização da parede abdominal de seus respectivos filhotes, quando o procedimento cirúrgico é realizado logo após o desmame, porque reduz a força máxima, a força de ruptura e a tensão na fase de cicatrização no $7^{\circ}$ dia, e no 21. ${ }^{\circ}$ dia de pós-operatório reduz a força de ruptura e a tensão. Faz-se necessário dar continuidade ao estudo, no sentido de identificar qual a influência deletéria da nicotina no processo de cicatrização das feridas.

\section{CONCLUSÃO}

De acordo com parâmetros tensiométricos, observouse efeito deletério da nicotina na cicatrização de feridas cirúrgicas em filhotes de ratas no período de gestação e lactação.

Alves MR, Campos ACL, Ioshii SO, Moraes-Jr H, Sakamoto D, Gortz LW. Nicotine effects during pregnancy and lactation on abdominal wall healing of young offspring rats: tensiometric study. ABCD Arq Bras Cir Dig 2009;22(2):110-4

ABSTRACT-Background - Smoking is deleterious to the healing process in the intra-womb fetus. Aim - To analyze the effects of nicotine, according with the tensiometric study (maximum strength, rupture strength and tensile strength), on the abdominal wall healing of suckling rats born of mothers that received nicotine, during pregnancy and lactation. Methods - Pregnant Wistar rats were randomly divided into two groups, the nicotine group (NG), in which it was administered subcutaneous nicotine at dose of $2 \mathrm{mg} / \mathrm{kg} /$ day, during pregnancy and lactation, and the control group (CG), that received subcutaneous isovolumetric saline solution at $0,9 \%$, in the same period of time. Sixty baby rats, divided into two groups, were weaned at 21 days and the transverse laparotomy was performed. Each group was divided into two subgroups, each one containing 15 baby rats, in accordance with the date when the surgical scar was analyzed: on the 7th day of postoperative, control subgroup C7 and nicotine subgroup N7, or 21st day of the postoperative, control subgroup C21 and nicotine subgroup N21. The surgical scar in the healing area was evaluated by tensiometric study. The statistics was based on the ANOVA model, at significance level of 5\%. Results - The tensiometric evaluation pointed out that the subgroup N7, in comparison to C7, presented lower maximum strength $(0,391 \pm 0,032 \mathrm{kgf}$ vs. $0,586 \pm 0,041 \mathrm{kgf}, P=0,0060)$, rupture strength $(0,393 \pm 0,032 \mathrm{kgf}$ vs. $0,577 \pm 0,051 \mathrm{kgf}, P=0,0047)$ and tensile strength $(0,029 \pm 0,002 \mathrm{kgf} / \mathrm{mm} 2$ vs. $0,036 \pm 0,005 \mathrm{kgf} / \mathrm{mm} 2, P=0,0493)$. The subgroup N21, when compared to $\mathrm{C} 21$, presented lower rupture strength $(0,421 \pm 0,004 \mathrm{kgf}$ vs. $0,581 \pm 0,002 \mathrm{kgf}, P=0,0007)$ and tensile strength $(0,053 \pm 0,002 \mathrm{kgf} / \mathrm{mm} 2$ vs. $0,066 \pm 0,002 \mathrm{kgf} / \mathrm{mm} 2, P=0,0056)$. Conclusion - Adverse effect of nicotine, administrated in pregnancy and lactation period, was observed on the abdominal wall healing of baby rats, according to the tensiometric parameter.

HEADINGS - Healing. Lactation. Laparotomy. Nicotine. Pregnancy. Tensiometry.

\section{REFERÊNCIAS}

1. Cabar FR, Carvalho FRC, Carvalho JP. Efeitos do tabagismo na saúde da mulher. Femina. 2003;31(4):373-75.

2. Chaib AS, Ling WS, Vasconcelos E. Avaliação experimental dos fios de sutura em um só plano. Rev Paul Med. 1972;80(5-6):217-26.

3. Forrest CR, Pang CY, Lindsay WK. Detrimental effect of nicotine on skin flap viability and blood flow in random skin flap on rats and pigs. Surg Forum. 1985;36:611-13

4. Forrest CR, Pang CY, Lindsay WK. Pathogenesis of ischemic necrosis in random-pattern skin flaps induced by long-term low-dose nicotine treatment in the rat. Plast Reconstr Surg. 1991;87(3):518-28.

5. Frick WG, Seals RR Jr. Smoking and wound healing: a review. Tex Dent J. 1994;111(6):21-3.

6. Harvey SC. The velocity of the growth of fibroblasts in the healing wound. Arch Surg. 1929;18(4):1227-40.

7. Horta BL, Victoria CG, Barros FC, Santos IS, Menezes AMB. Tabagismo em gestantes de área urbana da região sul do Brasil, 1982 e 1993. Rev Saude Publica. 1997:31:247-53.

8. Hughes PCR, Tanner JM. A longitudinal study of the growth of the blackhooded rat: methods of measurement and rates of growth for skull, limbs, pelvis, nose-rump and tail. J Anat. 1970; 106(2): 349-70.

9. Knolmayer TJ, Cornell KM, Bowyer MW, McCullough JS, Koenig W. Imbrication versus excision for fascial healing. Am J Surg. 1996;172(5):506-11.

10. Lehtovirta P, Forsj M. The acute effect of smoking on intervillous blood flow of the placenta. Br J Obstet Gynaecol. 1978;85:729-31.

11. Lindstedt E, Sandblom P. Wound healing in man: tensile strength of healing wounds in some patient groups. Ann Surg 1975; 181:842-846
12. Lymperopoulou A, Hainaut F, Crimal PH, Durana JL, Locatelli C, Maison C. Tabac et grossésse: recherché d'une correlation cotininemie et Doppler. J Gynecol Obstet Biol Reprod. 1996;25:824-7.

13. Maritz GS, Dennis H. Maternal nicotine exposure during gestation and lactation interferes with alveolar development in the neonatal lung. Reprod Fertil Dev. 1998;10(3):255-61.

14. Medeiros AC, Lima FP, Dantas Filho AM, Melo NMC, Azevedo IM. A nicotina atua como efeito deletério na reparação da parede abdominal. Acta Cir Bras. 2003;18(1):19-23.

15. Mosely LH, Finseth F. Cigarette smoking: impairment of digital blood flow and wound healing in the hand. The Hand. 1977;9(2):97-101.

16. Nash WA. Tração e compressão. In: Resistência dos materiais. São Paulo: Ed. McGraw-Hill do Brasil; 1972. p.11-20.

17. Orgill D, Demling RH. Current concepts and approaches to wound healing. Crit Care Med. 1988;16(9):899-907.

18. Richardson DR, Morton R, Howard J. Effects of chronic nicotine administration on RBC velocity in mesenteric capillaries of the rat. Blood Vessels. 1977;14(5):318-24.

19. Seid MH, Owens MM, Poole GV, Meeks GM. A randomized trial of abdominal incision suture technique and wound strength in rats. Arch Surg. 1995;130(4):394-7.

20. Skinivsky J, Malafaia O, Matias JEF, Ioshi SO, Chibayta M, Campos ACL, Czeczko NG. Nicotina interfere na cicatrização de anastomoses do intestino delgado em ratos. ABCD Arq Bras Cir Dig 2001; 14(4):

21. Van Winkle W, Hastings JC. Considerations in the choice of suture material for various tissues. Surg Gynecol Obstet. 1972;135(1):113-26.

Fonte de financiamento: não há

Conflito de interesse: não há

Recebido para publicação: 20/12/2008 Aceito para publicação: 03/03/2009 